\title{
Reduction in the risk of prostate cancer with finasteride
}

Prostate cancer is the most common cancer in men in both the UK and the USA. Although prostate cancer mortality has fallen due to prostate-specific antigen testing and improvements in therapeutic strategies, prostate cancer treatments are associated with adverse effects that can substantially impact quality of life. Active surveillance, the 'treatment' option often chosen by men with low-risk prostate cancer, is also associated with significant costs that add to the health-care burden for men with prostate cancer.

These factors make prostate cancer a prime target for chemoprevention. The role of finasteride, a $5 a$-reductase inhibitor, in the prevention of prostate cancer risk was investigated in the Prostate Cancer Prevention Trial (PCPT). Early results showed that 7 years of finasteride treatment reduced the relative risk of prostate cancer by $24.8 \%$, but was also associated with a relative increase of $26.9 \%$ in the risk of high-grade prostate cancers compared to treatment with placebo. Subsequent anlayses indicated that this increase in high-grade tumours with finasteride may have been due to detection bias but, nevertheless, this potential risk prevented the use of finasteride in prostate cancer chemoprevention.

\section{These results indicate that finasteride has a role in prostate cancer chemoprevention... 77}

Researchers, led by Ian Thompson, have now performed a follow-up analysis on PCPT participants, 18 years after the study began, to assess if finasteride is associated with an increased risk of death. Of 18,880 men who underwent randomization, $10.5 \%$ (989 of 9,423) were diagnosed with prostate cancer in the finasteride group and $14.9 \%(1,412$ of 9,457$)$ in the placebo group. In these patients, $3.5 \%$ in the finasteride group and $3.0 \%$ in the placebo group had high-grade cancer (Gleason score 7-10). Survival was similar between the two treatment groups: the 15-year survival rate was
$78.0 \%$ in the finasteride group compared with $78.2 \%$ in the placebo group. Survival rates were also comparable after 10 years, even when men were classified according to cancer grade: 10-year survival rate of men with low-grade prostate cancer was $83.0 \%$ in the finasteride group and $80.9 \%$ in the placebo group and $73.0 \%$ and $73.6 \%$, respectively, for men with high-grade cancer.

This study confirms that finasteride reduces prostate cancer risk and shows no significant difference between overall survival or survival after diagnosis of prostate cancer in those treated with placebo or finasteride. These results indicate that finasteride has a role in prostate cancer chemoprevention and could help to reduce the public health burden of the disease.

Vanessa Marchesi

Original article Thompson, I. M. Jr et al. Long-term survival of participants in the prostate cancer prevention trial. N. Engl. J. Med. 369, 603-610 (2013) 